\title{
Effect of osteoporosis on periodontal therapy among post- menopausal women
}

\author{
Isaac S. Gomes-Filho ${ }^{1}$, Tiago J. S. Oliveira ${ }^{1}$, Johelle S. Passos ${ }^{1}$, Eneida de M. M. Cerqueira ${ }^{2}$, \\ Simone S. da Cruz ${ }^{3}$, Maurício L. Barreto ${ }^{4}$, Julita M. F. Coelho ${ }^{2}$, Soraya C. Trindade ${ }^{1}$, \\ Carlos A. S. T. Santos ${ }^{5}$ and Viviane A. Sarmento ${ }^{1}$ \\ ${ }^{1}$ Department of Periodontics, Feira de Santana State University, Bahia, Brazil; ${ }^{2}$ Department of Biological Sciences, Feira de Santana State \\ University, Bahia, Brazil; ${ }^{3}$ Epidemiology Section, Federal University of Vale do São Francisco, Pernambuco, Brazil; ${ }^{4}$ Public Health Institute, \\ Federal University of Bahia, Salvador, Bahia, Brazil; ${ }^{5}$ Department of Statistics, Feira de Santana State University, Bahia, Brazil
}

doi: $10.1111 / j .1741-2358.2012 .00643 . x$

\section{Effect of osteoporosis on periodontal therapy among post-menopausal women}

Objective: This intervention study aimed to investigate the effect of osteoporosis on periodontal condition among 48 post-menopausal women undergoing periodontal therapy.

Material and methods: The experimental group, which underwent non-surgical periodontal therapy, was composed of 16 women with periodontitis to be treated, and the control group was formed by 32 women without periodontitis. Oral condition was assessed on three occasions: at the start of the treatment (first examination), 1 month (first re-examination) and 4 months after the end of the therapy (second re-examination). In the second re-examination, recurrence of periodontal disease was evaluated by comparing the clinical measurements obtained pre- and post-treatment. The diagnosis of osteoporosis was made by investigating densitometry reports obtained previously. Descriptive analysis, analysis of variance and the Bonferroni post hoc test were applied to the data gathered, with statistical significance level of $5 \%$. Results: The frequency of periodontitis was $50 \%$ in the treated group and $25 \%$ in the group without periodontitis. In both groups, this recurrence was greater in subjects with osteoporosis ( 37.5 and $18.75 \%$, respectively) than in the individuals without osteoporosis ( 12.5 and $6.25 \%$, respectively).

Conclusions: The preliminary results indicate that osteoporosis possibly has an influence on periodontal condition among individuals undergoing non-surgical periodontal treatment.

Keywords: osteoporosis, periodontal disease, periodontal medicine, menopause.

Accepted 25 September 2011

\section{Introduction}

Increasing longevity resulting from the epidemiological transition has had the implication that there is a need to take a new look at issues relating to the elderly population. There are diseases specific to this age group, and osteoporosis and periodontal disease are among them. These diseases notably eat up budgetary resources destined for healthcare.

Since the mid-1960s, the topic of the influence of osteoporosis on progression of periodontal disease has motivated the development of clinical studies in an attempt to elucidate this possible association, but the findings are still divergent and inconclusive.
Some of these studies have showed a lack of association $^{1-8}$ while others have revealed either a weak or a significant association ${ }^{9-17}$.

Osteoporosis is a multifactorial chronic disease characterised by reduction in bone mineral density (BMD) to below the minimum level required to ensure sufficient mechanical support, which increases its fragility and the risk of fractures ${ }^{18}$. It has been estimated that one in every four women at the menopause, and one in every three women beyond the age of 65 years, is affected by osteoporosis ${ }^{6}$. Data on its prevalence are scarce and discontinuous, but some surveys have estimated that it is present in 15-20\% of Brazilian women over the age of 50 years ${ }^{19}$. 
With regard to periodontitis, epidemiological studies have shown that, depending on the diagnostic criteria used, its worldwide prevalence is between 10 and $15 \%$, and it may reach $80 \%$ in certain regions ${ }^{20}$. It has more frequently been observed in older groups than in younger groups. Its multifactorial nature means that certain systematic conditions, including osteoporosis, appear to be additional predisposing factors.

In relation to periodontal treatment, osteoporosis is believed to still have an effect on the condition of the periodontal tissues after the therapy, given that it may affect the severity of the pre-existing periodontal disease ${ }^{2}$. Diminished bone density increases the susceptibility towards periodontal breakdown, and this can be understood through knowledge of the structural determinants of the bone tissue, which vary between individuals, between regions of the mouth and, more specifically, between tooth sites ${ }^{21}$.

However, this association is not established, which encourages the publication of studies still in preliminary stage. The justification for presenting preliminary results from this intervention study stems from the importance and great social relevance of the chronic diseases involved. The numbers of elderly people have been gradually increasing worldwide, along with the number of teeth preserved in their mouths. This situation indicates that increases in the two major public health problems of osteoporosis and periodontal disease will occur if they are not understood and if preventive measures are not taken ${ }^{22}$. From this perspective, this study had the aim of investigating the effect of osteoporosis on periodontal condition among post-menopausal women undergoing periodontal therapy, by means of an intervention study.

\section{Material and methods}

\section{Sample}

Of the 258 women initially eligible for this study, only 113 were selected after the use of exclusion criteria (Fig. 1).

A total of 113 women of minimum age 50 years who had been post-menopausal for at least 1 year were recruited from the diagnostic services for
Figure 1 Composition of the study sample among postmenopausal women. Feira de Santana, Bahia, Brazil, 2009. *Including 29 women living in other cities.

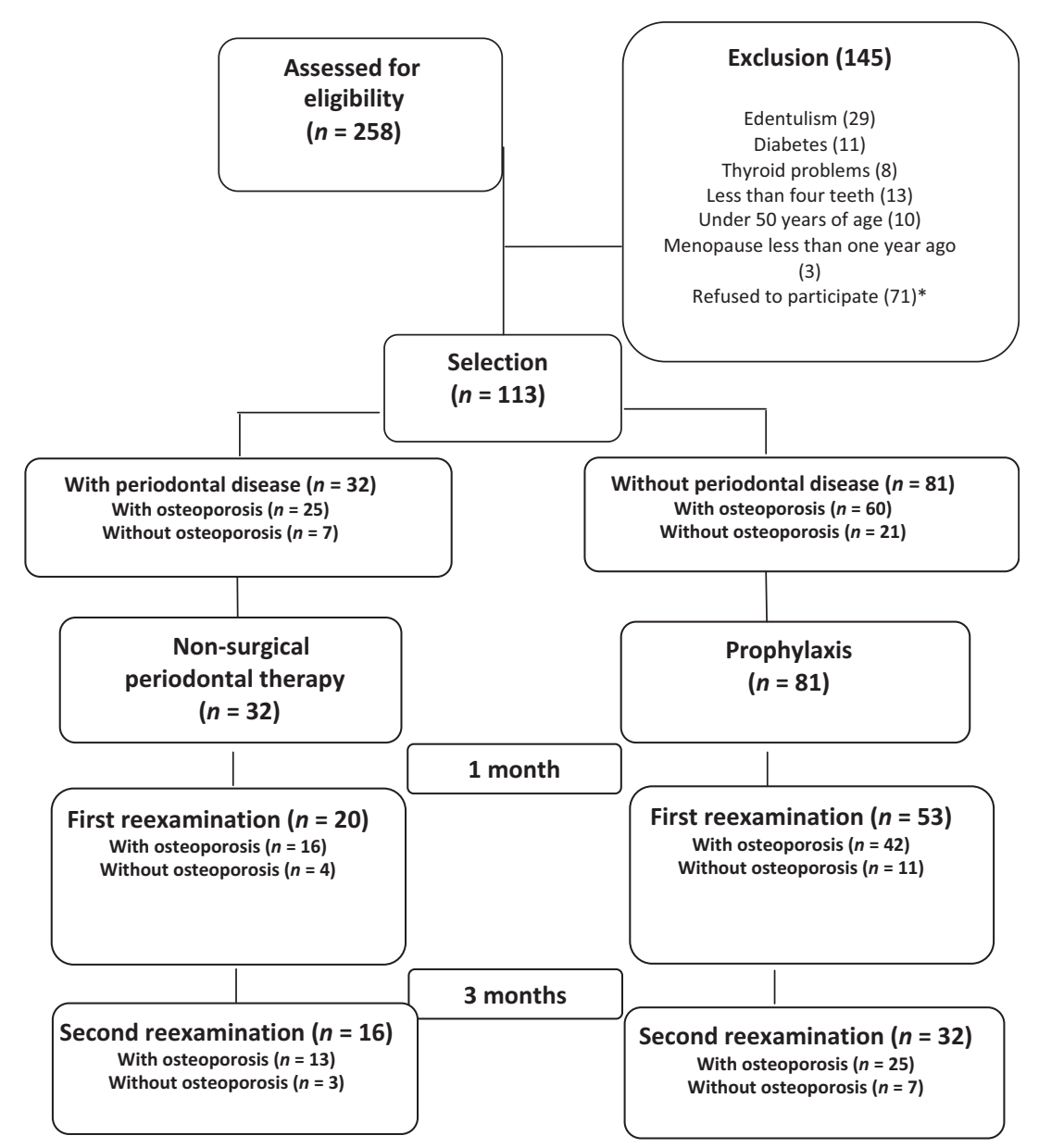


osteoporosis in Feira de Santana, Bahia, Brazil, to participate in this prospective study on post-menopausal women undergoing non-surgical periodontal therapy. All the participants signed a free and informed consent statement authorising their inclusion in the study, and all of them received assessments of their oral condition and the necessary dental treatment. This study was approved by the Ethics Committee of the Bahia Science Development Foundation, Salvador, Bahia, Brazil (protocol 047/2005).

The inclusion criteria were: (i) minimum age of 50 years; (ii) bone densitometry examination performed not more than 6 months earlier; (iii) postmenopausal status for at least 1 year; (iv) presence of at least four teeth in the mouth; and (v) absence of systemic conditions that might interfere with the inflammatory response, immune system and bone metabolism, such as kidney failure, diabetes or presence of bone lesions (tumour or osteomyelitis), observed in the dental arches.

Among the 113 women selected, only 48 were included in this study, given that by the time of this preliminary analysis, only these women had undergone reassessment 4 months after the nonsurgical periodontal therapy.

For this study, the experimental group was composed of 16 women with periodontitis to be treated and the control group was formed by 32 women without periodontitis.

\section{Data gathering procedures}

All the participants answered a questionnaire so that data relating to sociodemographic, biological and lifestyle factors could be obtained. They then underwent a clinical dental examination and were sent for a panoramic radiographic examination of the face to be performed, as a complementary evaluation on their oral condition. Densitometric reports on the participants were requested, and some information from these was extracted, such as the osteoporosis diagnosis, weight, height, BMD $\left(\mathrm{g} / \mathrm{cm}^{2}\right)$ and $t$-score.

A single examiner who, at the time of the examinations, was unaware of the BMD of the women under evaluation made all the clinical measurements on the both groups, throughout the study. Oral condition was assessed on three occasions: at the start of the treatment (first examination), 1 month after the end of the periodontal therapy (first re-examination) and 4 months after the end of the therapy (second re-examination). The reproducibility and concordance of the measurements were calculated by means of the intra-examiner kappa index, with regard to probing depth (0.6017) and recession/hyperplasia (0.6863). Inter-examiner measurements (comparison with the gold standard of an experienced examiner) were made in relation to probing depth $(0.6080)$ and recession/hyperplasia (0.6671).

\section{Periodontal clinical measurements}

Probing depth was obtained using a Williams probe(Hu-Friedy, Chicago, IL, USA) and was recorded at six locations for each tooth (mesiovestibular, mesiolingual, distovestibular, distolingual, midvestibular and mid-lingual) ${ }^{13}$. Likewise, the presence of bleeding on probing was determined as the rates measured at the above-mentioned six sites, by observing whether bleeding occurred within ten seconds after removing the probe from the pocket or sulcus. Clinical attachment measurements consisted of the summed values of the probing depth and gingival recession. Comparison between the clinical attachment measurements at the first examination and at the first and second re-examinations determined the criteria of clinical attachment loss, stability or gain.

At the first examination, the participants were deemed to have a diagnosis of periodontitis when they presented four or more teeth with one or more sites showing a probing depth $\geq 4 \mathrm{~mm}$ and a clinical attachment loss $\geq 3 \mathrm{~mm}$ at the same site, with bleeding present on stimulation. In threshold situations, in which the clinical descriptors came close to the criteria that had been established but were insufficient to conclude the diagnosis, radiographs were used to define the presence of periodontal disease. Thus, periodontitis was considered to be present when it was seen from the radiographic interpretation that there were four or more teeth with one or more sites showing periodontal bone reabsorption $\geq 3 \mathrm{~mm}$ apically, in relation to the cement-enamel junction. In this manner, the sample was divided into a group with periodontitis and another group without periodontitis.

\section{Periodontal therapy}

This consisted basically of non-surgical periodontal treatment for the women with periodontal disease (group with periodontitis), prophylaxis with oral hygiene instruction for the women without periodontal disease (group without periodontitis) and support therapy for the type of periodontal treatment that was received. The maximum number of sessions of root scaling and planning undertaken during the non-surgical periodontal treatment per 
participant was four sessions lasting a maximum of $\mathrm{l} \mathrm{h}$ each. It should be noted that complementary dental treatment was also provided, for example tooth extraction, endodontic treatment and fillings, among others. The support therapy consisted of prophylaxis. New oral hygiene instruction was applied, when this was seen to be necessary, in terms of the presence of calculi and large accumulations of plaque. The periodontal support therapy was continuous for all the participants.

\section{Diagnosis of recurrence of periodontal disease}

After the periodontal therapy, the condition of the periodontal tissues was assessed on two occasions: the first re-examination, 1 month after the end of the periodontal therapy; and the second re-examination, 4 months after the periodontal therapy. Comparison between the measurements obtained at these re-examinations characterised the periodontal disease in accordance with two recurrence criteria (RCs): 1.RCl, the criterion used for diagnosing periodontal disease at the first examination; 2. RC2, when the participant presented two or more teeth with one or more sites showing clinical attachment loss $\geq 2 \mathrm{~mm}$.

\section{Diagnosis of osteoporosis}

Dual-energy x-ray absorptiometry (DXA) was used to measure BMD in the regions of the lumbar spine and proximal femur in both groups (with and without periodontal disease). The BMD values were expressed as $T$-scores (standard deviation of the BMD value of the peak bone mass observed in young women). The presence of osteopenia was defined as a $T$-score between -1.0 and -2.0 , and osteoporosis as a $T$-score lower than $-2.5^{15}$. The diagnoses of osteoporosis and osteopenia were combined for analysis purposes. Thus, if osteopenia/osteoporosis was recorded in one of the two segments analyzed, the participant was considered to present osteoporosis. In this manner, the groups with and without periodontal disease were each divided into two subgroups: group with osteoporosis, composed of women with a diagnosis of osteopenia/osteoporosis; and group without osteoporosis, composed of women without this diagnosis.

\section{Data analysis procedures}

To describe the study population, the data were initially described according to the frequencies of sociodemographic, reproductive and lifestyle vari- ables, either pre-categorised based on their distribution or in accordance with an existing theoretical basis in the literature. Continuous variables were expressed as their mean values and standard deviations, and Student's $t$ test was applied to determine any statistically significant differences in the oral clinical variables between the groups with and without periodontitis, at the start of the study and 4 months after the treatment $(p<0.05)$. The frequency of recurrence of periodontitis according to the criteria RCl and RC2 was described in the groups with and without osteoporosis by means of Fisher's test.

Analysis of variance (ANOVA) for repeated measurements, followed by the Bonferroni post hoc test, was used to compare the periodontal clinical changes that occurred between the first examination and the first re-examination (1 month after periodontal therapy or dental prophylaxis) and between the first examination and the second re-examination (4 months after the periodontal therapy or dental prophylaxis) in the groups with and without periodontitis, stratified for osteoporosis. The significance level was exchange to $\alpha=0.05$.

The sPss statistical software was used for the data analysis (version 10; Chicago, IL, USA).

\section{Results}

Table 1 shows that the mean age among the postmenopausal women was $59.2 \pm 6.8$ years, the mean age at the menarche was $13.6 \pm 1.8$ years and the mean age at the menopause was $47.0 \pm 6.4$ years. Natural menopause was the most common type among the individuals in this sample $(65.2 \%)$. Most of the participants in this study were non-smokers $(92 \%)$ and not alcohol consumers (76.8\%), 74.3\% had attended school for $<4$ years, $83 \%$ had an income greater than one minimum monthly salary and $82.3 \%$ had black or mixed skin colour. Among the other characteristics, these were seen in a little more than $50 \%$, according to the cut-off point established for dichotomisation of the variable.

As expected, Table 2, the group with periodontitis presented statistically significant differences between the periodontal clinical descriptors obtained from the first examination and those from the second re-examination, consequent to the effect of the therapy used. Even in the group without periodontitis, which only received dental prophylaxis and oral hygiene motivation, statistically significant differences in the above-mentioned periodontal clinical descriptors were noted, with the exception of the clinical attachment level $(p>0.05)$. 
Table 1 Sociodemographic, reproductive and lifestyle characteristics among postmenopausal women. Feira de Santana, Bahia, Brazil, $2009(n=113)^{\mathrm{a}}$.

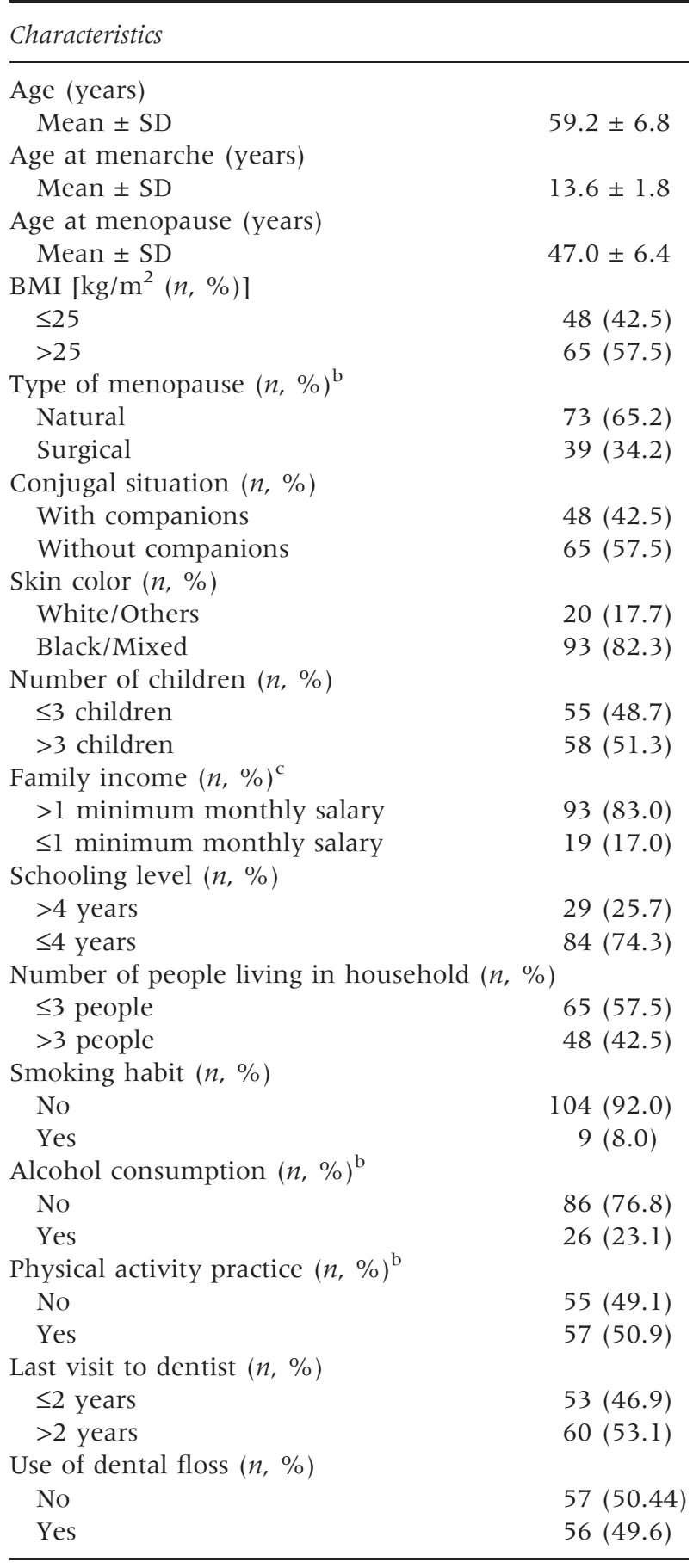

${ }^{a}$ Sample with all the women who formed part of the intervention study, independent of not yet having been monitored for completion of the short-term investigation period.

${ }^{\mathrm{b}} \mathrm{A}$ data was missing.

${ }^{\mathrm{c}}$ Value of the minimum salary: R\$ 46500 (US\$ 278 944), at the time of data gathering.
There were also statistically significant decreases in the mean numbers of teeth with probing depth $\geq 4 \mathrm{~mm}$ and clinical attachment level $\geq 5 \mathrm{~mm}$, also because of the direct effect of the treatment and monitoring of the periodontal conditions in the group with treated periodontitis. With regard to the mean number of teeth with a clinical attachment level of $1-2 \mathrm{~mm}$, a statistically significant increase was recorded over the 4-month period, both in the group with and in the group without periodontitis. On the other hand, there was no statistical difference in the mean number of teeth present. Finally, the mean number of teeth with a clinical attachment level of 3-4 mm did not present any statistically significant difference between the start of the therapy and 4 months after the therapy.

According to Table 3, the analysis between the groups on the percentage of the teeth with a clinical attachment level of 3-4 mm, there were significant differences between the first examination and the first re-examination $(p=0.019)$, and between the first and second re-examinations $(p=0.010)$, for the group with periodontitis.

Four months after periodontal therapy, the frequencies of recurrence of periodontal disease were evaluated. With regard to $\mathrm{RCl}$, it was observed only one case of periodontitis after treatment, among individuals with normal BMD, and that recurrence was not recorded among those without osteoporosis. The frequency of periodontitis according to RC2 was $50 \%$ in the group treated of periodontitis and $25 \%$ in the group without periodontitis (Fig. 2). In both groups, this recurrence was greater in subjects with osteoporosis ( 37.5 and $18.75 \%$, respectively) than in the individuals with normal BMD (12.5 and $6.25 \%$, respectively).

\section{Discussion}

The main preliminary findings from this study indicate that osteoporosis may have an influence on the periodontal condition of post-menopausal women undergoing periodontal therapy, considering that the recurrence of periodontitis was more frequent among the individuals with osteoporosis. Literature on the topic of the influence of osteoporosis on periodontal condition is scarce, with only a few studies indicating that such a relationship may exist ${ }^{9-17}$.

As this study, some investigations assessed the average measurements of periodontal clinical descriptors, comparing the distribution of these 
Table 2 Distribution of oral health conditions before (first examination) and 4 months after periodontal therapy (second reexamination) among the postmenopausal women with and without a diagnosis of periodontitis. Feira de Santana, Bahia, Brazil, $2009(n=48)^{\mathrm{a}}$.

\begin{tabular}{|c|c|c|c|c|c|c|}
\hline \multirow[b]{2}{*}{ Characteristics } & \multicolumn{3}{|c|}{ Group with periodontitis $(n=16)$} & \multicolumn{3}{|c|}{ Group without periodontitis $(n=32)$} \\
\hline & $\begin{array}{l}\text { First } \\
\text { examination }\end{array}$ & $\begin{array}{l}\text { Second } \\
\text { reexamination }\end{array}$ & $p^{\mathrm{b}}$ & $\begin{array}{l}\text { First } \\
\text { examination }\end{array}$ & $\begin{array}{l}\text { Second } \\
\text { reexamination }\end{array}$ & $p^{\mathrm{b}}$ \\
\hline \multicolumn{7}{|c|}{ Probing depth (mm) } \\
\hline Mean \pm SD & $2.4 \pm 0.5$ & $2.0 \pm 0.4$ & $<0.001$ & $1.9 \pm 0.3$ & $1.7 \pm 0.2$ & 0.020 \\
\hline \multicolumn{7}{|c|}{ Clinical attachment level (mm) } \\
\hline Mean \pm SD & $3.4 \pm 0.8$ & $2.9 \pm 0.8$ & $<0.001$ & $2.4 \pm 0.5$ & $2.4 \pm 0.4$ & 0.127 \\
\hline \multicolumn{7}{|c|}{ Bleeding on probing $(\%)$} \\
\hline Mean \pm SD & $28.1 \pm 19.2$ & $6.6 \pm 4.0$ & $<0.001$ & $14.6 \pm 12.6$ & $5.4 \pm 4.1$ & $<0.001$ \\
\hline \multicolumn{7}{|c|}{ Plaque index $(\%)$} \\
\hline Mean \pm SD & $35.6 \pm 30.3$ & $11.4 \pm 11.4$ & 0.009 & $22.7 \pm 23.2$ & $7.1 \pm 8.8$ & $<0.001$ \\
\hline \multicolumn{7}{|c|}{ Number of teeth present } \\
\hline Mean \pm SD & $13.4 \pm 5.7$ & $13.2 \pm 5.8$ & 0.456 & $13.0 \pm 5.5$ & $13.0 \pm 5.5$ & 0.161 \\
\hline \multicolumn{7}{|c|}{$\%$ Teeth with clinical attachment level of $1-2 \mathrm{~mm}$} \\
\hline Mean \pm SD & $6.2 \pm 10.4$ & $13.9 \pm 14.5$ & 0.031 & $17.6 \pm 20.0$ & $26.9 \pm 24.4$ & 0.012 \\
\hline \multicolumn{7}{|c|}{$\%$ Teeth with clinical attachment level of 3-4 mm } \\
\hline Mean \pm SD & $39.4 \pm 26.6$ & $39.7 \pm 25.3$ & 0.954 & $59.4 \pm 23.9$ & $53.1 \pm 21.0$ & 0.153 \\
\hline \multicolumn{7}{|c|}{$\%$ Teeth with clinical attachment level $\geq 5 \mathrm{~mm}$} \\
\hline Mean \pm SD & $53.4 \pm 29.9$ & $45.6 \pm 34.9$ & 0.043 & $12.4 \pm 11.3$ & $10.3 \pm 12.7$ & 0.317 \\
\hline \multicolumn{7}{|c|}{$\%$ Teeth with probing depth $\geq 4 \mathrm{~mm}$} \\
\hline Mean \pm SD & $27.5 \pm 24.9$ & $10.0 \pm 12.4$ & $<0.001$ & $5.0 \pm 11.7$ & $2.3 \pm 4.8$ & 0.214 \\
\hline
\end{tabular}

${ }^{a}$ Final sample that underwent the periodontal treatment and was monitored until reaching 4 months after the treatment. Women who did not reach this length of short-term follow-up in this study were excluded.

${ }^{\mathrm{b}} p$ Value; statistical significance: $p \leq 0.05$.

measures, according to the presence of osteoporosis. In these investigations, were found significant differences in mean measurements of clinical attachment ${ }^{14,23,24}$, probing depth ${ }^{3,25}$ and percentage of gingival bleeding ${ }^{10}$ between groups with and without osteoporosis. These studies reinforce the findings found here as they defend the existence of the correlation between osteoporosis and periodontal disease.

On the other hand, other studies have been unable to demonstrate the possible influence of changes in BMD on periodontal tissues ${ }^{1-8}$.

The findings of this study should be evaluated carefully, first of all because they are not definitive and form part of the preliminary results from a 12-month follow-up study. Nonetheless, some methodological precautions were taken to ensure greater credibility for the interpretation, such as in relation to sample selection, choosing the exposure and outcome measurements and standardising the examiner and data analysis procedures.

Thus, the diagnostic criteria for osteoporosis used in this study were the criteria standardised by the World Health Organization ${ }^{18}$. With regard to the diagnosis of periodontal disease, an association of four periodontal clinical descriptors was used with aim of including individuals in the experimental group who truly had periodontal disease ${ }^{26}$, thereby avoiding diagnostic bias through using a criterion of good specificity.

It is important to make it clear that, in accordance with the robust outcome measurement used for the initial diagnosis in this study, thus defining groups with and without periodontitis, greater evidence that all the participants in the group to be treated truly had a diagnosis of periodontal disease was sought, without including possible false positives. In this way, the group without periodontitis incorporated women with mild levels of periodontal disease. This could be an initial explanation for the high occurrence (recurrence) of periodontal disease in this group at the follow-up 4 months after the periodontal therapy. Moreover, it was evident that this occurred in higher proportions among the women with abnormal BMD (osteoporosis).

Another point to be noted is the positive result from the periodontal treatment, reinforced through analysis comparing the periodontal condition before the treatment or prophylaxis and 4 months afterwards. It was evident that there was an improvement in the periodontal clinical 
Table 3 Distribution of periodontal clinical conditions at the first examination (before the treatment), first reexamination ( 1 month after the treatment) and second reexamination (4 months after the treatment) among postmenopausal women with and without osteoporosis. Feira de Santana, Bahia, Brazil, $2009(n=48)^{\mathrm{a}}$.

\begin{tabular}{|c|c|c|c|c|}
\hline & $\begin{array}{l}\% \text { Teeth with } \\
\text { clinical attachment } \\
\text { level of } 1-2 \mathrm{~mm}\end{array}$ & $\begin{array}{l}\% \text { Teeth with } \\
\text { clinical attachment } \\
\text { level of 3-4 mm }\end{array}$ & $\begin{array}{l}\% \text { Teeth with } \\
\text { clinical attachment } \\
\text { level }>5 \mathrm{~mm}\end{array}$ & $\begin{array}{l}\% \text { Probing } \\
\text { depth }>4 \mathrm{~mm}\end{array}$ \\
\hline \multicolumn{5}{|c|}{ With periodontitis and with normal BMD $(n=3)$} \\
\hline First examination & $7.7 \pm 13.3$ & $40.4 \pm 37.8^{\mathrm{a}}$ & $51.9 \pm 41.7^{\mathrm{C}}$ & $44.5 \pm 23.3^{\mathrm{ef}}$ \\
\hline First reexamination & $15.2 \pm 9.4$ & $59.4 \pm 14.4^{\mathrm{ab}}$ & $25.4 \pm 17.3^{\mathrm{cd}}$ & $18.4 \pm 22.0^{\mathrm{f}}$ \\
\hline Second reexamination & $23.6 \pm 20.6$ & $25.7 \pm 29.8^{\mathrm{b}}$ & $50.7 \pm 46.2^{\mathrm{d}}$ & $22.6 \pm 21.5^{\mathrm{e}}$ \\
\hline \multicolumn{5}{|c|}{ With periodontitis and with osteoporosis $(n=13)$} \\
\hline First examination & $11.6 \pm 13.1$ & $39.1 \pm 25.4^{\mathrm{a}}$ & $53.7 \pm 28.8^{c}$ & $23.6 \pm 24.4^{\mathrm{ef}}$ \\
\hline First reexamination & $17.2 \pm 8.1$ & $45.6 \pm 29.7^{\mathrm{ab}}$ & $46.4 \pm 34.7^{\mathrm{c}}$ & $9.4 \pm 9.5^{\mathrm{f}}$ \\
\hline Second reexamination & $19.7 \pm 7.6$ & $42.8 \pm 24.3^{\mathrm{b}}$ & $44.4 \pm 34.1$ & $7.1 \pm 8.1^{\mathrm{e}}$ \\
\hline \multicolumn{5}{|c|}{ Without periodontitis and with normal BMD $(n=7)$} \\
\hline First examination & $32.8 \pm 19.2$ & $70.0 \pm 24.8$ & $11.2 \pm 19.4$ & $5.9 \pm 6.6$ \\
\hline First reexamination & $33.9 \pm 17.2$ & $66.7 \pm 31.2$ & $13.9 \pm 31.3$ & $3.9 \pm 5.8$ \\
\hline Second reexamination & $42.3 \pm 24.9$ & $57.0 \pm 25.8$ & $11.7 \pm 25.5$ & $3.7 \pm 4.9$ \\
\hline \multicolumn{5}{|c|}{ Without periodontitis and with osteoporosis $(n=25)$} \\
\hline First examination & $22.8 \pm 20.3$ & $56.4 \pm 23.3$ & $25.6 \pm 21.3$ & $4.7 \pm 12.8$ \\
\hline First reexamination & $28.8 \pm 18.8$ & $55.1 \pm 20.5$ & $23.7 \pm 23.8$ & $1.1 \pm 3.0$ \\
\hline Second reexamination & $33.3 \pm 23.6$ & $52.0 \pm 20.0$ & $22.2 \pm 20.0$ & $1.2 \pm 4.7$ \\
\hline
\end{tabular}

$\mathrm{BMD}$, bone mineral density.

${ }^{a}$ Final sample that underwent the periodontal treatment and was monitored until reaching 4 months after the treatment. Women who did not reach this length of short-term follow-up in this study were excluded.

The same letters represent the groups that differed statistically after the Bonferroni post-hoc test $(p \leq 0.05)$.

${ }^{\mathrm{a}} p=0.019 ;{ }^{\mathrm{b}} p=0.010 ;{ }^{\mathrm{c}} p=0.011 ;{ }^{\mathrm{d}} p=0.015 ;{ }^{\mathrm{e}} p=0.013 ;{ }^{\mathrm{f}} p=0.011$.

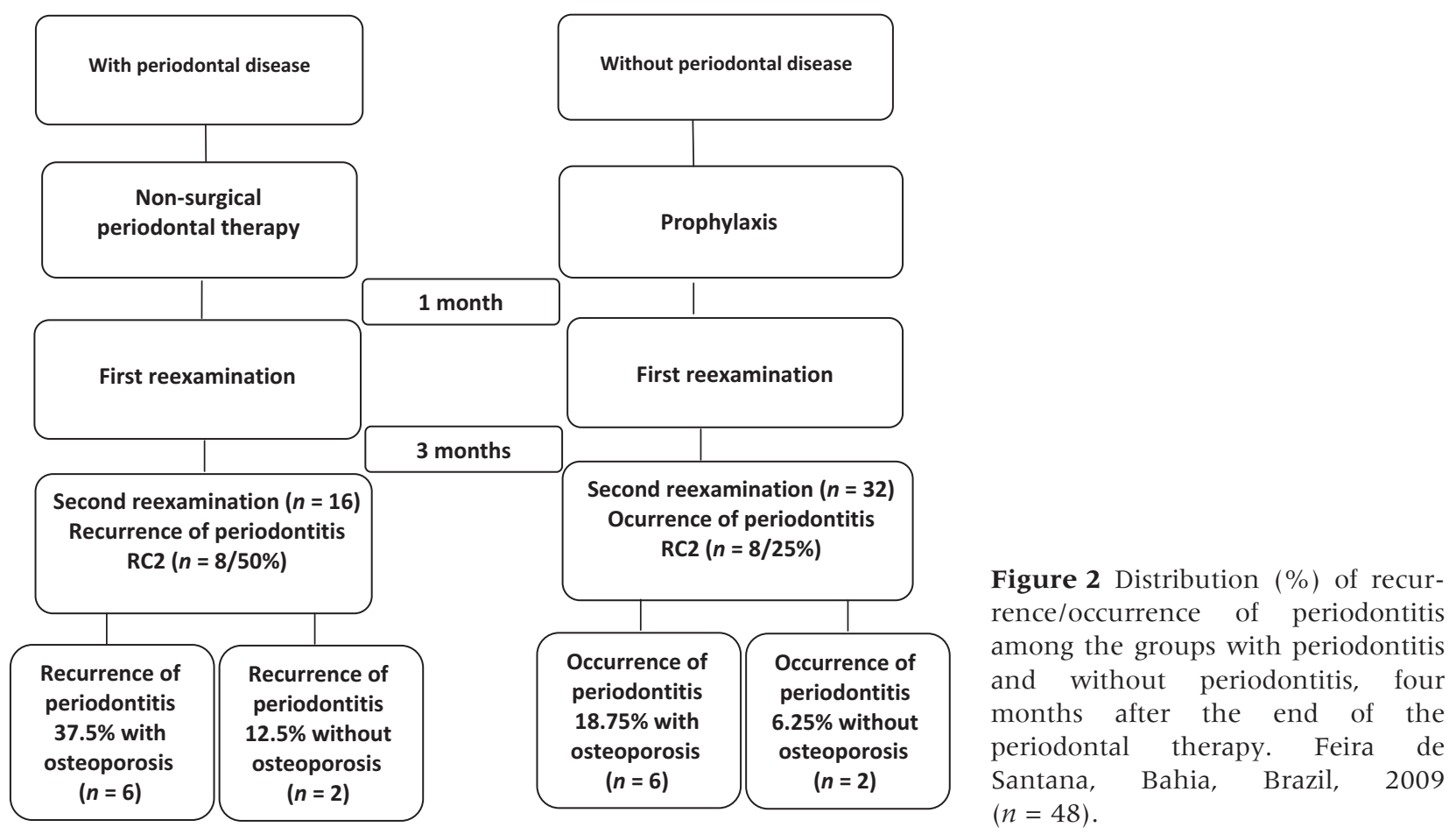

descriptors in both groups over the period of the study, reaching statistical significance. Thus, the results from this study corroborate classic studies

in the literature regarding the effect from nonsurgical periodontal treatment, on periodontal tissues $^{27}$. 
Finally, follow-up on the study participants over a 12-month study period, along with empowerment of this investigation, may provide greater elucidation of this matter.

\section{Conclusions}

From this perspective, and contributing towards the body of evidence on this topic, these preliminary findings indicate that osteoporosis may have an influence on periodontal condition, although definitive information will be gathered through completing the 12 months of follow-up on these women, given that this investigation is currently in progress. Furthermore, it can be emphasised that for future evaluations on the topic of the influence of osteoporosis on periodontal condition, it will be important to use analytical procedures with notable control variables such as hormonal therapy and smoking habit, among others that were not considered in this preliminary descriptive study, along with analysis on the doseresponse effect at different levels of severity of periodontal disease.

\section{Acknowledgements}

To the National Council for Scientific and Technological Development (CNPq), the Research Support Foundation of the State of Bahia (FAPESB) and Feira de Santana State University for financial support for the research. Thanks also to all the people who contributed towards the investigation. The authors declare that they do not have any conflicts of interest relating to this study.

\section{References}

1. Kribbs PJ. Comparison of mandibular bone in normal and osteoporotic women. J Prosthet Dent 1990; 63: 218-222.

2. Hildebolt CF, Pilgram TK, Dotson $M$ et al. Attachment loss with postmenopausal age and smoking. J Periodontal Res 1997; 32: 619-625.

3. Weyant RJ, Pearlstein ME, Churak AP et al. The association between osteopenia and periodontal attachment loss in older women. J Periodontol 1999; 70: 982-991.

4. Pilgram TK, Hildebolt CF, Dotson M et al. Relationships between clinical attachment level and spine and hip bone mineral density: data from healthy postmenopausal women. J Periodontol 2002; 73: 298301.

5. Famili P, Cauley J, Suzuki JB et al. Longitudinal study of periodontal disease and edentulism with rates of bone loss in older women. J Periodontol 2005; 76: $11-15$.

6. Pallos D, Ceschin A, Victor GdeA et al. Menopause: a risk factor for periodontal disease? (in Portuguese). Rev Bras Ginecol Obstet 2006; 28: 292-297.

7. Mafetano LR, Souza DM, Perozini C et al. Influence the levels of bone mineral density in alveolar bone loss and periodontal clinical parameters in postmenopausal women (in Portuguese). Rev Assoc Med Rio Grande Do Sul 2007; 51: 280-284.

8. Sultan N, Rao J. Association between periodontal disease and bone mineral density in postmenopausal women: a cross sectional study. Med Oral Patol Oral Cir Bucal 2011; 16: e440-e447.

9. Von Wowern N, Klausen B, Kollerup G. Osteoporosis: a risk factor in periodontal disease. J Periodontol 1994; 65: 1134-1138.

10. Payne JB, Reinhardt RA, Nummikoski PV et al. Longitudinal alveolar bone loss in postmenopausal osteoporotic/osteopenic women. Osteoporos Int 1999; 10: $34-40$.

11. Tezal M, Wactawski-Wende J, Grossi SG et al. The relationship between bone mineral density and periodontitis in postmenopausal women. J Periodontol 2000; 71: 1492-1498.

12. Wactawski-Wende $\mathbf{J}$, Hausmann E, Hovey $\mathbf{K}$ et al. The association between osteoporosis and alveolar crestal height in postmenopausal women. $J$ Periodontol 2005; 76: 2116-2124.

13. Gomes-Filho IS, Passos Jde S, Cruz SS et al. The association between postmenopausal osteoporosis and periodontal disease. J Periodontol 2007; 78: 17311740 .

14. Lopes FF, Loureiro FHF, Pereira AFV et al. Bone mineral density vs clinical periodontal status: cross sectional study in post menopausal women (in Portuguese). Rev Assoc Med Bras 2008; 54: 41 1-414.

15. Nicopoulou-Karayianni K, Tzoutzoukos P, Mitsea A et al. Tooth loss and osteoporosis: the osteodent study. J Clin Periodontol 2009; 36: 190-197.

16. Al Habashneh $\mathbf{R}$, Alchalabi H, Khader YS et al. Association between periodontal disease and osteoporosis in postmenopausal women in Jordan. J Periodontol 2010; 81: 1613-1621.

17. Renvert S, Berglund J, Persson RE et al. Osteoporosis and periodontitis in older subjects participating in the Swedish National Survey on Aging and Care (SNAC-Blekinge). Acta Odontol Scand 2011; 69: 201-207.

18. World Health Organization. Assessment of fracture risk and its application to screening for postmenopausal osteoporosis. Report of a WHO Study Group. World Health Organ Tech Rep Ser 1994; 843: 1129.

19. Costa-Paiva L, Horovitz AP, Santos AO et al. Prevalence of osteoporosis in post menopausal women and association with clinical and reproductive factors (in Portuguese). Rev Bras Ginecol Obstet 2003; 25: 507-512. 
20. Kinane DF, Peterson M, Stathopoulou G. Environmental and other modifying factors of the periodontal disease. Periodontol 2000 2006; 40: 107-119.

21. Gomes-Filho IS, Sarmento VA, de Castro MS et al. Radiographic features of periodontal bone defects: evaluation of digitized images. Dentomaxillofac Radiol 2007; 36: 256-262.

22. Yoshihara A, Seida Y, Hanada N et al. A longitudinal study of the relationship between periodontal disease and bone mineral density in communitydwelling older adults. J Clin Periodontol 2004; 31: 680684.

23. Erdogan Ö, Incki KK, Benlidayi ME et al. Dental and radiographic findings as predictors of osteoporosis in postmenopausal women. Geriatr Gerontol Int 2009; 9: 155-164.

24. Brennan RM, Genco RJ, Hovey KM et al. Clinical attachment loss, systemic bone density, and subgingival calculus in postmenopausal women. J Periodontol 2007; 78: 2104-2111.
25. Shen EC, Gau CH, Hsieh YD et al. Periodontal status in post-menopausal osteoporosis: a preliminary clinical study in Taiwanese women. J Chin Med Assoc 2004; 67: 389-393.

26. Gomes-Filho IS, Cruz SS, Rezende EJ et al. Exposure measurement in the association between periodontal disease and prematurity/low birth weight. J Clin Periodontol 2007; 34: 957-963.

27. Badersten A, Niveus R, Egelberg J. 4-year observations of basic periodontal therapy. $J$ Clin Periodontol 1987; 14: 438-444.

Correspondence to:

Isaac S. Gomes-Filho - Avenida Getúlio Vargas, 379, Centro. Feira de Santana, Bahia 44.025-010, Brazil.

Tel: 557536230661

Fax: 557536230661

E-mail: isuzart@gmail.com 